\title{
Pharmacokinetic Concentration Specimen Type
}

National Cancer Institute

\section{Source}

National Cancer Institute. Pharmacokinetic Concentration Specimen Type. NCI

Thesaurus. Code C87967.

The type of a material sample taken from a biological entity for pharmacokinetic concentration testing. 